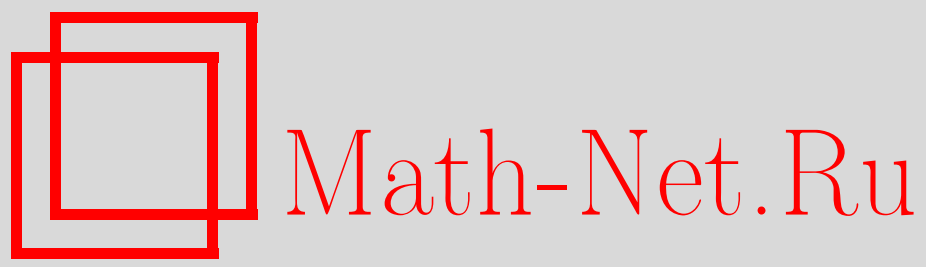

М. С. Агранович, Сильно эллиптические системы 2-го порядка с граничными условиями на незамкнутой липшицевой поверхности, Функи. анализ и его прил., 2011, том 45, выпуск 1, 1-15

DOI: https://doi.org/10.4213/faa3031

Использование Общероссийского математического портала MathNet.Ru подразумевает, что вы прочитали и согласны с пользовательским соглашением http://www. mathnet.ru/rus/agreement

Параметры загрузки:

IP : 52.6 .47 .48

26 апреля 2023 г., 13:14:52

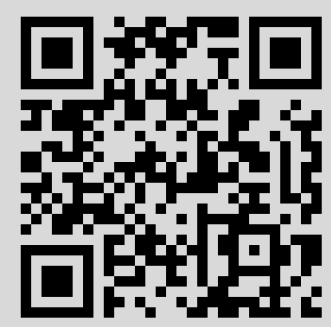


Функциональный анализ и его приложения

2011, т. 45, вып. 1, с. 1-15

УДК $517.98+517.95$

\title{
Сильно эллиптические системы 2-го порядка с граничными условиями на незамкнутой липшицевой поверхности*
}

\author{
(C) 2011. М. С. АГРАнович
}

\begin{abstract}
Рассматриваются граничные задачи и задачи сопряжения для сильно эллиптических систем 2-го порядка с граничными условиями на компактной незамкнутой липшицевой поверхности $S$ с липшицевым краем. Основная цель - выяснение условий однозначной разрешимости этих задач в пространствах $H^{s}-$ простейших $L_{2}$-пространствах типа Соболева - с использованием операторов типа потенциала на $S$. Обсуждаются вопросы о регулярности решений с выходом в несколько более общие пространства бесселевых потенциалов и Бесова и о свойствах решений спектральных задач со спектральным параметром в условиях сопряжения на $S$, включая асимптотики собственных значений.
\end{abstract}

1. Постановки задач. Мы рассмотрим сильно эллиптическую систему 2-го порядка для простоты на $n$-мерном торе $\mathbb{T}=\mathbb{T}^{n}$ с $2 \pi$-периодическими координатами $x_{j}(j=1, \ldots, n)$ вне $(n-1)$-мерной липшицевой поверхности $S$ с $(n-2)$-мерным липшицевым краем $\partial S(n \geqslant 2)$. Более точно, мы будем считать, что $S$ - часть замкнутой липшицевой поверхности $\Gamma$, делящей тор на две области $\Omega^{ \pm}$, и что граница $\partial S$ делит $\Gamma$ на две области, $S=S_{1}$ и $S_{2}$. В выборе дополнительной части $S_{2}$ границы $Г$ имеется очевидный произвол. Система задана на всем торе и записана в дивергентной форме:

$$
L u:=-\sum \partial_{j} a_{j, k}(x) \partial_{k} u(x)+\sum b_{j}(x) \partial_{j} u(x)+c(x) u(x)=f(x) .
$$

Здесь коэффициенты - это $m \times m$-матрицы с комплексными элементами и $u-$ вектор-столбец высоты $m ; a_{j, k} \in C^{1}(\mathbb{T}), b_{j} \in C^{0,1}(\mathbb{T})$ (липшицевы) и $c \in L_{\infty}(\mathbb{T})$; $\partial_{k}=\partial / \partial x_{k}$. Стороны границы $\Gamma$, обращенные к $\Omega^{ \pm}$, обозначим через $\Gamma^{ \pm}$. Аналогично определим $S^{ \pm}$. Граничные условия будут задаваться на $S^{ \pm}$.

Через $H^{s}$ мы обозначаем простейшие $L_{2}$-пространства бесселевых потенциалов; при $s \geqslant 0$ это $L_{2}$-пространства Соболева-Слободецкого. Все обозначения и предположения, кроме наличия края у граничной поверхности, такие же, как в [3]. Сводка сведений об используемых в настоящей статье пространствах $H^{s}$, $H_{p}^{s}$ и $B_{p}^{s}$ содержится в [3]; в обзоре [4] она повторена и немного дополнена. Можно смотреть также [5]. (Все работы автора, включенные в литературу, можно найти на его странице в Интернете http://www.agranovich.nm.ru.)

Сильная эллиптичность - это равномерная положительная определенность действительной части главного символа - матрицы $a(x, \xi)=\sum a_{j, k}(x) \xi_{j} \xi_{k}$ при вещественных $\xi,|\xi|=1$. Дополнительно действительная часть скалярного произведения $(c u, u)_{\mathbb{T}}$ предполагается достаточно большой - настолько, что

* Поддержано грантом РФФИ. 
отвечающая системе форма

$$
\Phi_{\mathbb{T}}(u, v)=\int_{\mathbb{T}}\left[\sum a_{j, k}(x) \partial_{k} u(x) \cdot \partial_{j} \bar{v}(x)+\sum b_{j}(x) u(x) \cdot \bar{v}(x)+c(x) u(x) \cdot \bar{v}(x)\right] d x
$$

коэрцитивна на пространстве $H^{1}(\mathbb{T})$ в смысле справедливости (усиленного) неравенства Гординга $C \operatorname{Re} \Phi_{\mathbb{T}}(u, u) \geqslant\|u\|_{H^{1}(\mathbb{T})}^{2}$. Как следствие уравнение $L u=f$ однозначно разрешимо в $H^{1}(\mathbb{T})$ при $f \in H^{-1}(\mathbb{T})$ в силу леммы Лакса-Мильграма о слабых решениях абстрактного уравнения $L u=f$, где $L$ - ограниченный оператор, определяемый приведенной ниже формулой (1.4). Приведем формулировку этой леммы в нужной нам форме (ср., например, [21]).

Лемма 1.1. Пусть $H$ - гильбертово пространство и $H^{*}$ - сопряженное $\kappa$ нему пространство относительно формь $(f, v), v \in H, f \in H^{*}, u$ пусть задано $f \in H^{*}$. Предположим, что для непрерьвной полуторалинейной формы $\Phi(u, v)$ на $H$ выполнено неравенство

$$
C \operatorname{Re} \Phi(u, u) \geqslant\|u\|_{H}^{2}
$$

Тогда существует один и только один элемент и $\in$, такой, что

$$
\Phi(u, v)=(f, v)
$$

при всех $v \in H, u$ оператор $L^{-1}: f \mapsto u$ ограничен.

Далее, аналогичные форме $\Phi_{\mathbb{T}}(u, v)$ формы $\Phi_{\Omega^{ \pm}}(u, v)$ коэрцитивны на пространствах $\widetilde{H}^{1}\left(\Omega^{ \pm}\right)$, у нас в смысле справедливости неравенств $C \operatorname{Re} \Phi_{\Omega^{ \pm}}(u, u) \geqslant$ $\|u\|_{\widetilde{H}^{1}\left(\Omega^{ \pm}\right)}^{2}$. (Через $\widetilde{H}^{s}\left(\Omega^{ \pm}\right)$обозначаются подпространства в $H^{s}(\mathbb{T})$, состоящие из элементов с носителями в $\overline{\Omega^{ \pm}}$.) Этим обеспечивается однозначная разрешимость задач Дирихле в $\Omega^{ \pm}$. Коэрцитивность форм $\Phi_{\Omega^{ \pm}}$на $H^{1}\left(\Omega^{ \pm}\right)$дополнительно предполагаем; известны достаточные для этого условия. Она обеспечивает однозначную разрешимость задач Неймана в $\Omega^{ \pm}$. В частности, можно рассматривать обобщенные системы неоднородной анизотропной упругости (см., например, [25]) и уравнение Бельтрами-Лапласа с младшими членами.

Пусть $\Omega_{0}=\mathbb{T} \backslash \bar{S}$. Обратим внимание на то, что эта область не является липшицевой. Нам нужно определить пространство $H^{1}\left(\Omega_{0}\right)$. Было бы неправильно определять его как пространство сужений функций из $H^{1}(\mathbb{T})$ на $\Omega_{0}$, так как при таком определении следы функций из него на $S^{ \pm}$всегда были бы одинаковыми. Определим $H^{1}\left(\Omega_{0}\right)$ как пространство функций $u$ из $L_{2}\left(\Omega_{0}\right)$, сужения которых на $\Omega^{ \pm}$принадлежат $H^{1}$, со следами $\gamma^{ \pm} u=u^{ \pm}$на $\Gamma^{ \pm}$(они принадлежат $\left.H^{1 / 2}(\Gamma)\right)$, совпадающими на $S_{2}$; при этом

$$
\|u\|_{H^{1}\left(\Omega_{0}\right)}^{2}=\|u\|_{H^{1}\left(\Omega^{+}\right)}^{2}+\|u\|_{H^{1}\left(\Omega^{-}\right)}^{2} .
$$

Отсюда следует, что скачок $[u]=u^{-}-u^{+}$на $S$ принадлежит $\widetilde{H}^{1 / 2}(S)$. (Через $\widetilde{H}^{s}(S)$ обозначается подпространство элементов в $H^{s}(\Gamma)$ с носителями в $\bar{S}$.)

Замечание. Это определение пространства $H^{1}\left(\Omega_{0}\right)$ равносильно следующему стандартному определению. Это пространство состоит из таких принадлежащих $L_{2}$ в области $\Omega_{0}$ функций $u$, что все их первые производные $\partial_{j} u$ в смысле обобщенных функций в этой области тоже принадлежат $L_{2}$; при этом

$$
\|u\|_{H^{1}\left(\Omega_{0}\right)}^{2}=\|u\|_{L_{2}\left(\Omega_{0}\right)}^{2}+\sum\left\|\partial_{j} u\right\|_{L_{2}\left(\Omega_{0}\right)}^{2} .
$$


Равносильность следует из формулы Нечаса интегрирования по частям для функций из $H^{1}$ в липшицевой области [24, с. 121]: если написать ее в $\Omega^{ \pm}$для функции $u$, принадлежащей $H^{1}\left(\Omega_{0}\right)$ в смысле первого определения, и основной функции из $C_{0}^{\infty}\left(\Omega_{0}\right)$, то после сложения этих формул члены на $S_{2}$ сокращаются.

Следовательно, пространство $H^{1}\left(\Omega_{0}\right)$ не зависит от выбора поверхности $S_{2}$.

Поскольку система $L u=f$ на торе предположена однозначно разрешимой, будем считать, что $f=0$ в $\Omega_{0}$.

Решения граничных задач для системы $L u=0$ ищутся в $H^{1}\left(\Omega_{0}\right)$ (до п. 6 мы будем рассматривать только простейшие пространства). Это означает, в частности, что если $\varphi-$ любая функция из $C_{0}^{\infty}\left(\Omega_{0}\right)$, то $(u, \widetilde{L} \varphi)_{\Omega_{0}}=0$. Здесь $\widetilde{L}-$ оператор, формально сопряженный к $L$.

Мы рассмотрим следующие задачи.

$1^{\circ}$. Задача Дирихле для системы $L u=0$ в $\Omega_{0}$ с условиями Дирихле на $S^{ \pm}$:

$$
u^{ \pm}=g^{ \pm} \text {на } S^{ \pm},
$$

где $g^{ \pm} \in H^{1 / 2}(S)$ и $[g]=g^{-}-g^{+} \in \widetilde{H}^{1 / 2}(S)$.

$2^{\circ}$. Задача Неймана для той же системы в $\Omega$ с условиями Неймана

$$
T^{ \pm} u=h^{ \pm} \text {на } S^{ \pm},
$$

где $T^{ \pm} u$ - конормальная производная, $h^{ \pm} \in H^{-1 / 2}(S)$ и $[h] \in \widetilde{H}^{-1 / 2}(S)$.

Поясним последнюю постановку. Напомним, что конормальная производная $T^{ \pm} u$ функции $u$ из $H^{1}\left(\Omega^{ \pm}\right)$в общем случае определяется по $u$ и $L u=f$ формулой Грина

$$
(f, v)_{\Omega^{ \pm}}=\Phi_{\Omega^{ \pm}}(u, v) \mp\left(T^{ \pm} u, v^{ \pm}\right)_{\Gamma} .
$$

См. [21, с. 117]. Здесь $v-$ любая пробная функция из $H^{1}\left(\Omega^{ \pm}\right)$и $f \in \widetilde{H}^{-1}\left(\Omega^{ \pm}\right)$, в формах слева и справа используется соответствующая двойственность. Конормальная производная оказывается принадлежащей $H^{-1 / 2}(\Gamma)$. Значит, она принадлежит $H^{-1 / 2}(S)$ и то же верно для скачка $[T u]=T^{-} u-T^{+} u$. Однако справедливо следующее

Предложение 1.2. Пусть и - решение системы (1.1) в $\Omega_{0}$, принадлежащее $H^{1}\left(\Omega_{0}\right)$. Тогда $[T u] \in \widetilde{H}^{-1 / 2}(S)$, m. e. $\operatorname{supp}[T u] \subset \bar{S}$.

Доказательство. Если $f=L u \in L_{2}\left(\Omega^{ \pm}\right)$, то $u$ можно рассматривать как элемент пространства $E\left(\Omega^{ \pm}\right)$с нормой

$$
\|u\|_{E\left(\Omega^{ \pm}\right)}=\left(\|u\|_{H^{1}\left(\Omega^{ \pm}\right)}^{2}+\|L u\|_{L_{2}\left(\Omega^{ \pm}\right)}^{2}\right)^{1 / 2} .
$$

В этом пространстве плотно пространство $C^{\infty}\left(\overline{\Omega^{ \pm}}\right)$сужений на $\Omega^{ \pm}$функций из $C^{\infty}(\mathbb{T})$. (В $[18$, с. 59] это по существу проверено для лапласиана, но доказательство сохраняется в общем случае.) На гладких функциях конормальная производная вычисляется по формуле

$$
T^{ \pm} u=\sum \nu_{j}(x) a_{j, k}(x) \gamma^{ \pm} \partial_{k} u(x),
$$

где $\nu_{j}(x)$ - координаты единичной нормали к $Г$ (она есть почти во всех точках $x \in \Gamma)$, которую считаем направленной в $\Omega^{-} ; \gamma^{ \pm} w=w^{ \pm}-$оператор перехода к следу. При аппроксимации в $E\left(\Omega^{ \pm}\right)$гладкими функциями имеет место сходимость конормальных производных в $H^{-1 / 2}(\Gamma)$. (Сp. [22], где рассмотрены более 
общие $f$.) В частности, у нас $f=0$ и конормальные производные аппроксимирующих функций одинаковы с двух сторон вблизи любой точки на $S_{2}$. Значит, носитель скачка $[T u]$ лежит на $\bar{S}$.

В акустике и электродинамике поверхность с краем - модель незамкнутого экрана, в теории упругости - модель трещины. В этих случаях подобные задачи рассматривали Стефан и Костабель-Стефан (см., в частности, [28]-[30], [12]). $\mathrm{У}$ них задачи упругости рассмотрены для системы Ламе (изотропная среда). Кроме того, $g^{ \pm}$первоначально считались совпадающими и $h^{ \pm}-$тоже. Обобщения на анизотропные среды в трехмерных областях получены, в частности, в работах Дудучавы-Натрошвили-Шаргородского [15] и Дудучавы-Вендланда [16]. См. также [19] и литературу в этих работах. Во всех этих работах поверхность $S$ и ее край предполагались достаточно гладкими и применялся метод ВинераХопфа с использованием псевдодифференциальных операторов в форме, предложенной Эскиным в [17]. Это позволило не только изучить регулярность решения, но и исследовать его асимптотическое поведение при приближении к краю поверхности $S$. В этих работах просматривается возможность обобщений более простых результатов об однозначной разрешимости (или фредгольмовости) на произвольные сильно эллиптические системы 2-го порядка в липшицевых областях без предположений о гладкости, без псевдодифференциальных операторов и без метода Винера-Хопфа. Именно эта возможность реализуется в настоящей работе. Используется тот же путь сведе́ния задач к эквивалентным уравнениям на $S$. Автор затрагивал эти вопросы в [6], но там общность недостаточна. Конечно, без метода Винера-Хопфа до асимптотики решений вряд ли можно добраться, но небольшое продвижение в вопросе о регулярности решений мы все же получим, и оно полезно при нахождении асимптотики собственных значений спектральных задач, постановка которых указана ниже (задачи $5^{\circ}, 6^{\circ}$ ).

Мы рассмотрим также следующие задачи для системы $L u=0$ в $\Omega_{0}$.

$3^{\circ}$. Задача с условиями

$$
[u]=g, \quad[T u]=h \text { на } S .
$$

Здесь $g \in \widetilde{H}^{1 / 2}(S), h \in \widetilde{H}^{-1 / 2}(S)$.

$4^{\circ}$. Смешанная задача с условиями

$$
u^{+}=g \text { на } S^{+}, \quad T^{-} u=h \text { на } S^{-} .
$$

Здесь снова $g \in H^{1 / 2}(S), h \in H^{-1 / 2}(S)$. При рассмотрении этой задачи мы следуем работе Дудучавы-Натрошвили [14], в которой рассмотрена система анизотропной упругости, но снова обходимся без предположений гладкости и без метода Винера-Хопфа. Здесь мы предполагаем систему (1.1) формально самосопряженной. Сp. [21, с. 231-234], где рассмотрены общие смешанные задачи в липшицевой области с замкнутой границей и получены эквивалентные уравнения на ней в случае формально самосопряженной системы.

Литература по смешанным задачам чрезвычайно обширна, задача $4^{\circ}$ является нестандартным вариантом этих задач. В [5] автор рассмотрел общие (стандартные) смешанные задачи для сильно эллиптических систем 2-го порядка в липшицевой области с замкнутой границей и получил эквивалентные уравнения на ней без предположения о формальной самосопряженности. Там можно проследить некоторые параллели с настоящей работой. 
$5^{\circ}$. Первая спектральная задача

$$
[u]=0, \quad u^{ \pm}=-\lambda[T u] \text { на } S .
$$

$6^{\circ}$. Вторая спектральная задача

$$
[T u]=0, \quad[u]=-\lambda T^{ \pm} \text {на } S .
$$

Подобные спектральные задачи в случае замкнутой липшицевой границы рассмотрены в [3], [4]. Их первоначальная постановка в случае уравнения Гельмгольца принадлежит московскому физику Б. З. Каценеленбауму и его сотрудникам Н. Н. Войтовичу и А. Н. Сивову, см. [31] или [8].

2. Операторы типа потенциала и задача $3^{\circ}$. Оператор $L^{-1}$, обратный к оператору $L$ на торе, является интегральным оператором, это так называемый ньютонов потенциал. Его ядро $\mathscr{E}(x, y)$ - фундаментальное решение для $L$. Напомним, что потенциалы простого и двойного слоя определяются на заданных на Г функциях (с небольшим запасом регулярности) формулами

$$
\begin{aligned}
& \mathscr{A} \psi(x)=\int_{\Gamma} \mathscr{E}(x, y) \psi(y) d S_{y}, \\
& \mathscr{B} \varphi(x)=\int_{\Gamma}\left(\widetilde{T}_{y}^{+} \mathscr{E}^{*}(x, y)\right)^{*} \varphi(y) d S_{y} .
\end{aligned}
$$

Здесь $\widetilde{T}^{+}(\cdot)$ - конормальная производная для формально сопряженного к $L$ оператора $\widetilde{L}$ (см. [21]). Исследование этих операторов элементарными средствами проведено в [21]; см. также [3]. Нам понадобится ряд проверенных там утверждений об этих операторах. В частности, их можно найти в [3]. Перечислим их.

1. Оператор $\mathscr{A}$ продолжается до оператора, действующего ограниченным образом из $H^{-1 / 2}(\Gamma)$ в $H^{1}(\mathbb{T})$ и, значит, в $H^{1}\left(\Omega^{ \pm}\right)$. Функция $\mathscr{A} \psi$ при $\psi \in H^{-1 / 2}(\Gamma)$ является решением системы $L u=0$ в $\Omega^{ \pm}$. След $A \psi=\gamma^{ \pm} \mathscr{A} \psi$ этой функции на $\Gamma$ - ограниченный оператор из $H^{-1 / 2}(\Gamma)$ в $H^{1 / 2}(\Gamma)$. При предположении о коэрцитивности форм $\Phi_{\Omega^{ \pm}}$на $\widetilde{H}^{1}\left(\Omega^{ \pm}\right)$оператор $A$ обратим.

2. Оператор $\mathscr{B}$ действует ограниченным образом из $H^{1 / 2}(\Gamma)$ в $H^{1}\left(\Omega^{ \pm}\right)$. Функция $\mathscr{B} \varphi$ при $\varphi \in H^{1 / 2}(\Gamma)$ является решением системы $L u=0$ в $\Omega^{ \pm}$.

3. Положим, как в [21],

$$
B=\frac{1}{2}\left(\gamma^{+} \mathscr{B}+\gamma^{-} \mathscr{B}\right) \quad \text { и } \quad \widehat{B}=\frac{1}{2}\left(T^{+} \mathscr{A}+T^{-} \mathscr{A}\right) .
$$

Первый из этих операторов - это прямое значение потенциала двойного слоя, он ограничен в $H^{1 / 2}(\Gamma)$. Второй ограничен в $H^{-1 / 2}(\Gamma)$ и равен $\widetilde{B}^{*}$ : является сопряженным к прямому значению потенциала двойного слоя для $\widetilde{L}$ относительно продолжения стандартного скалярного произведения в $L_{2}(\Gamma)$ на прямое произведение $H^{1 / 2}(\Gamma) \times H^{-1 / 2}(\Gamma)$. При нашем предположении о коэрцитивности форм $\Phi_{\Omega^{ \pm}}$на $H^{1}\left(\Omega^{ \pm}\right)$операторы $\frac{1}{2} I \pm B$ и $\frac{1}{2} I \pm \widehat{B}$ обратимы.

4. Справедливы соотношения

$$
T^{ \pm} \mathscr{A}= \pm \frac{1}{2} I+\widehat{B}, \quad \gamma^{ \pm \mathscr{B}}=\mp \frac{1}{2} I+B, \quad T^{+} \mathscr{B}=T^{-} \mathscr{B} .
$$

Оператор $H=-T^{ \pm} \mathscr{B}-$ это так называемый гиперсингулярный оператор, он действует ограниченным образом из $H^{1 / 2}(\Gamma)$ в $H^{-1 / 2}(\Gamma)$. При нашем предположении о коэрцитивности форм $\Phi_{\Omega^{ \pm}}$на $H^{1}\left(\Omega^{ \pm}\right)$оператор $H$ обратим. Оператор 
$H^{-1}$ связан с $A$ соотношениями

$$
H^{-1}=\left(\frac{1}{4} I-B^{2}\right)^{-1} A=A\left(\frac{1}{4} I-\widehat{B}^{2}\right)^{-1} .
$$

5. Формула

$$
u=\mathscr{B} \varphi-\mathscr{A} \psi, \quad \text { где } \varphi=[u], \quad \psi=[T u],
$$

дает представление решения системы $L u=0$ в $\Omega^{ \pm}$через скачки на $Г$. Здесь $u \in H^{1}\left(\Omega^{ \pm}\right), \varphi \in H^{1 / 2}(\Gamma), \psi \in H^{-1 / 2}(\Gamma)$.

Этот перечень мы будем дополнять по мере надобности.

Теперь перейдем к случаю поверхности $S$ с краем. В этом случае формулу (2.6) можно записать со скачками на $S$ :

$$
u=\mathscr{B}[u]_{S}-\mathscr{A}[T u]_{S} .
$$

Эта функция - решение системы $L u=0$ вне $\bar{S}$, принадлежащее $H^{1}\left(\Omega_{0}\right)$, так как оно принадлежит $H^{1}\left(\Omega^{ \pm}\right)$и $u^{+}=u^{-}$на $S_{2}$. Как нетрудно видеть, получается

Предложение 2.1. Задача $3^{\circ}$ однозначно разрешима, и формула (2.7) въражает ее решение.

3. Задачи Дирихле и Неймана. Введем операторы

$$
A_{S} \psi=\left.(A \psi)\right|_{S}, \quad B_{S} \varphi=\left.(B \varphi)\right|_{S} \quad\left(\psi \in \widetilde{H}^{-1 / 2}(S), \varphi \in \widetilde{H}^{1 / 2}(S)\right) .
$$

Очевидно, что $A_{S}$ - ограниченный оператор из $\widetilde{H}^{-1 / 2}(S)$ в $H^{1 / 2}(S)$, а $B_{S}-$ ограниченный оператор из $\widetilde{H}^{1 / 2}(S)$ в $H^{1 / 2}(S)$.

Рассмотрим задачу Дирихле. Переходя в (2.7) с двух сторон на $S$, складывая получающиеся соотношения и деля на 2 , получаем

$$
g=B_{S} \varphi-A_{S} \psi, \quad \text { где } g=\frac{1}{2}\left(g^{+}+g^{-}\right), \varphi=g^{-}-g^{+} .
$$

Это уравнение относительно $\psi$, аналогичное использованным в работах [28][30], [12], [15], [16].

Предложение 3.1. Для оператора $A_{S}$ справедливо неравенство типа Гординга

$$
\|\psi\|_{\widetilde{H}^{-1 / 2}(S)}^{2} \leqslant C_{1} \operatorname{Re}\left(A_{S} \psi, \psi\right)_{S}, \quad \psi \in \widetilde{H}^{-1 / 2}(S)
$$

u, следовательно, этот оператор обратим в силу леммы Лакса-Мильграма.

Поясним, что справа используется двойственность пространств $H^{1 / 2}(S)$ и $\widetilde{H}^{-1 / 2}(S)$ относительно продолжения стандартного скалярного произведения в $L_{2}(S)$ на прямое произведение этих пространств. Неравенство (3.3) вытекает из аналогичного неравенства для оператора $A$ :

$$
\|\psi\|_{H^{-1 / 2}(\Gamma)}^{2} \leqslant C_{1} \operatorname{Re}(A \psi, \psi)_{\Gamma}, \quad \psi \in H^{-1 / 2}(\Gamma),
$$

последнее влечет обратимость этого оператора в силу той же леммы. В [3] вывод этого неравенства опущен, проверено аналогичное неравенство для операторов $N_{ \pm}$(Neumann-to-Dirichlet). Для полноты изложения проверим неравенство (3.4).

Для $u=\mathscr{A} \psi$ из формул Грина (1.9) в $\Omega^{ \pm}$в силу соотношения $\psi=-[T \mathscr{A} \psi]$ (см. первое равенство в (2.4)) получается формула

$$
\Phi_{\Omega^{+}}(u, u)+\Phi_{\Omega^{-}}(u, u)=(\psi, A \psi)_{\Gamma} .
$$


Из (усиленных) неравенств Гординга в $\Omega^{ \pm}$получаем

$$
\|u\|_{H^{1}\left(\Omega^{+}\right)}^{2}+\|u\|_{H^{1}\left(\Omega^{-}\right)}^{2} \leqslant C_{2} \operatorname{Re}(\psi, A \psi)_{\Gamma}=C_{2} \operatorname{Re}(A \psi, \psi)_{\Gamma} .
$$

Так как задачи Неймана у нас предположены однозначно разрешимыми, а априорные оценки для их решений в случае однородной системы являются двусторонними, то

$$
\left\|T^{ \pm} u\right\|_{H^{-1 / 2}(\Gamma)} \leqslant C_{3}\|u\|_{H^{1}\left(\Omega^{ \pm}\right)} .
$$

Значит,

$$
\|\psi\|_{H^{-1 / 2}(\Gamma)}^{2}=\|[T \mathscr{A} \psi]\|_{H^{-1 / 2}(\Gamma)}^{2} \leqslant C_{1} \operatorname{Re}(A \psi, \psi)_{\Gamma} .
$$

Неравенство (3.4) проверено.

Теорема 3.2. Задача Дирихле в постановке $1^{\circ}$ имеет одно и только одно решение, принадлежсащее $H^{1}\left(\Omega_{0}\right)$.

Действительно, скачок $[T u]_{S}$ определяется по данным Дирихле из уравнения (3.2), после чего решение строится по формуле (2.7). При нулевых данных Дирихле это решение - нулевое.

Перейдем к задаче Неймана. Здесь картина аналогична. Введем оператор

$$
H_{S} \varphi=\left.(H \varphi)\right|_{S} \quad\left(\varphi \in \widetilde{H}^{1 / 2}(S)\right) .
$$

Это ограниченный оператор из $\widetilde{H}^{1 / 2}(S)$ в $H^{-1 / 2}(S)$. Вычислив конормальные производные от обеих частей формулы (2.7) с двух сторон на $S$, сложив и разделив на 2, получим уравнение относительно $\varphi$

$$
h=-H_{S} \varphi-\widehat{B}_{S} \psi, \text { где } h=\frac{1}{2}\left(h^{+}+h^{-}\right), \quad \psi=h^{-}-h^{+},
$$

оно аналогично использованным в тех же работах [28]-[30], [12], [15], [16].

Предложение 3.3. Для оператора $H_{S}$ справедливо неравенство типа Гординга

$$
\|\varphi\|_{\widetilde{H}^{1 / 2}(S)}^{2} \leqslant C_{4} \operatorname{Re}\left(H_{S} \varphi, \varphi\right)_{S}, \quad \varphi \in \widetilde{H}^{1 / 2}(S),
$$

так что этот оператор обратим в силу леммы Лакса-Мильграма.

Здесь справа используется двойственность пространств $H^{-1 / 2}(S)$ и $\widetilde{H}^{1 / 2}(S)$. Неравенство (3.7) вытекает из аналогичного неравенства для оператора $H$ :

$$
\|\varphi\|_{H^{1 / 2}(\Gamma)}^{2} \leqslant C_{4} \operatorname{Re}(H \varphi, \varphi)_{\Gamma}, \quad \varphi \in H^{1 / 2}(\Gamma) .
$$

Для доказательства последнего неравенства полагаем $u=\mathscr{B} \varphi$ и выводим из формул Грина соотношение

$$
\Phi_{\Omega^{+}}(u, u)+\Phi_{\Omega^{-}}(u, u)=(H \varphi, \varphi)_{\Gamma} .
$$

Далее действуем, как в предыдущем случае, пользуясь тем, что оценки для решений задач Дирихле являются двусторонними.

В неравенствах (3.4), (3.3), (3.8), (3.7) проявляется хорошо известная идея о «сильной эллиптичности» операторов типа потенциала на границе, отвечающих сильно эллиптическим системам. См. указания и литературу в [20].

Теперь аналогично теореме 3.2 получается

Теорема 3.4. Задача Неймана в постановке $2^{\circ}$ имеет одно и только одно решение, принадлежсащее $H^{1}\left(\Omega_{0}\right)$.

Отметим, что аналогичные операторам $A_{S}$ и $H_{S}$ операторы $N_{S}$ и $D_{S}$ рассматриваются в [5]. 
4. Смешанная задача. Чтобы построить ее решение $u$, будем искать скачки

$$
\varphi=[u]_{S} \in \widetilde{H}^{1 / 2}(S), \quad \psi=[T u]_{S} \in \widetilde{H}^{-1 / 2}(S) .
$$

Вычисляя конормальную производную функции $(2.7)$ на $S^{-}$и граничное значение этой функции на $S^{+}$, получаем уравнения

$$
\begin{aligned}
-H_{S} \varphi-\left(-\frac{1}{2} I+\widehat{B}_{S}\right) \psi & =h, \\
\left(-\frac{1}{2} I+B_{S}\right) \varphi-A_{S} \psi & =g .
\end{aligned}
$$

Уравнения не случайно записаны в этом порядке: мы следуем [14] и [21]. Оператор

$$
\mathscr{T}=\left(\begin{array}{cc}
H_{S} & -\frac{1}{2} I+\widehat{B}_{S} \\
\frac{1}{2} I-B_{S} & A_{S}
\end{array}\right)
$$

действует ограниченным образом из пространства

$$
\widetilde{\mathscr{H}}=\widetilde{H}^{1 / 2}(S) \times \widetilde{H}^{-1 / 2}(S)
$$

в пространство

$$
\mathscr{H}=H^{-1 / 2}(S) \times H^{1 / 2}(S) .
$$

Эти два пространства двойственны относительно продолжения скалярного произведения $\left(u_{1}, v_{1}\right)_{S}+\left(u_{2}, v_{2}\right)_{S}$ на их прямые произведения. Для столбца $U=$ $(\varphi, \psi)^{\prime}$ имеем

$$
(\mathscr{T} U, U)=\left(H_{S} \varphi, \varphi\right)_{\Gamma}+\left(\left(-\frac{1}{2} I+\widehat{B}_{S}\right) \psi, \varphi\right)_{\Gamma}+\left(\left(\frac{1}{2} I-B_{S}\right) \varphi, \psi\right)_{\Gamma}+\left(A_{S} \psi, \psi\right)_{\Gamma} .
$$

Предположим, что $L-$ формально самосопряженный оператор. В это предположение мы включаем условие

$$
\sum b_{j} \nu_{j}=0 \text { на } S,
$$

см. [4]. Тогда $\widehat{B}=B^{*}$ и из (4.6) видно, что

$$
\operatorname{Re}(\mathscr{T} U, U)=\left(H_{S} \varphi, \varphi\right)_{\Gamma}+\left(A_{S} \psi, \psi\right)_{\Gamma},
$$

остальное сокращается (знак Re справа сейчас не нужен). Воспользуемся предложениями 3.1 и 3.3. Получаем, что уравнение $\mathscr{T} U=F$, где $F-$ столбец $(h, g)^{\prime}$, однозначно разрешимо по лемме Лакса-Мильграма. Этим доказана

Теорема 4.1. При условии формальной самосопряженности оператора $L$ задача $4^{\circ}$ однозначно разрешима.

Можно рассмотреть случай, когда только главная часть оператора $L$ является формально самосопряженной, но на этом не будем останавливаться.

5. Спектральные задачи. Для задач на собственные функции справедлиBO

Предложение 5.1. Задачи $5^{\circ}$ и $6^{\circ}$ эквивалентны соответственно уравнениям

$$
A_{S} \psi=\lambda \psi, \quad \text { əде } \psi=[T u]_{S}, u=-\mathscr{A} \psi,
$$

$u$

$$
H_{S}^{-1} \varphi=\lambda \varphi, \quad \text { əде } \varphi=[u]_{S}, u=\mathscr{B} \varphi .
$$

Проверка аналогична проведенной в [3] в случае поверхности без края. 
Здесь можно пояснить, что обратимые операторы $A_{S}: \widetilde{H}^{-1 / 2}(S) \rightarrow H^{1 / 2}(S)$ и $H_{S}: \widetilde{H}^{1 / 2}(S) \rightarrow H^{-1 / 2}(S)$ действуют из более широкого пространства в более узкое в первом случае и наоборот во втором; промежуточным является, в частности, пространство $L_{2}(S)$. Поэтому спектральные задачи имеют смысл.

Далее мы приводим спектральные результаты, аналогичные изложенным в [4] в случае границы Г без края, поэтому не будем останавливаться на некоторых подробностях.

Рассмотрим оператор $A_{S}$. Наиболее благоприятным является случай формально самосопряженной системы (1.1). В этом случае $\left(A_{S} \psi_{1}, \psi_{2}\right)_{S}$ - скалярное произведение в $\widetilde{H}^{-1 / 2}(S)$, относительно которого $A_{S}$ является самосопряженным оператором с положительным дискретным спектром. Из собственных функций составляется ортонормированный базис, и он остается ортогональным базисом в $H^{1 / 2}(S)$ относительно скалярного произведения $\left(A_{S}^{-1} \varphi_{1}, \varphi_{2}\right)_{S}$. Этот результат переносится на промежуточные пространства $H^{s}(S)=\widetilde{H}^{s}(S)$, $|s|<1 / 2$. Для собственных значений $\lambda_{j}$ оператора $A_{S}$, упорядоченных в порядке невозрастания с учетом кратностей, справедлива оценка $\lambda_{j} \leqslant C j^{-1 /(n-1)}$. Более того, получается асимптотическая формула для собственных значений: в п. 7 мы укажем путь к ее получению.

Если лишь главная часть оператора $L-$ формально самосопряженный оператор, то $A_{S}$ - слабое возмущение самосопряженного оператора $A_{S}^{0}$, который строится по «подправленной» в младших членах системе $L_{0} u=0$. Собственные значения содержатся в сколь угодно узком угле с биссектрисой $\mathbb{R}_{+}$с некоторого номера. Система корневых функций полна в $\widetilde{H}^{-1 / 2}(S)$ и $H^{1 / 2}(S)$ и является там базисом для метода суммирования Абеля-Лидского со скобками порядка $n-1+\varepsilon$ со сколь угодно малым $\varepsilon>0$. Результат о полноте переносится на промежуточные пространства.

Наконец, в самом общем случае спектр дискретен и сохраняется оценка $s$-чисел $s_{j} \leqslant C_{1} j^{-1 /(n-1)}$. Полнота и суммируемость методом Абеля-Лидского сохраняются, если раствор угла с биссектрисой $\mathbb{R}_{+}$, содержащего все значения форм $\Phi_{\Omega^{ \pm}}(u, u)$, меньше $\pi /(n-1)$. Собственные значения лежат в этом угле. Для доказательства в дополнительном угле выводится оптимальная оценка для резольвенты оператора $A_{S}^{-1}$ : если (буквы $\varphi$ и $\psi$ раньше имели другой смысл)

$$
\left(A_{S}^{-1}-\lambda I\right) \varphi=\psi
$$

TO

$$
\|\varphi\|_{H^{1 / 2}(S)}+|\lambda|\|\varphi\|_{\widetilde{H}^{-1 / 2}(S)} \leqslant C_{2}\|\psi\|_{\widetilde{H}^{-1 / 2}(S)} \cdot
$$

См. указания в [3, п. 6]. Снова результат о полноте переносится на промежуточные пространства.

Спектральные свойства оператора $H_{S}^{-1}$ аналогичны только что изложенным. Если $L$ - формально самосопряженный оператор, то в $H^{-1 / 2}(S)$ вводится скалярное произведение $\left(H_{S}^{-1} \psi, \psi\right)_{\Gamma}$.

\section{6. Регулярность решений.}

6.1. Мы продолжаем считать формы $\Phi_{\Omega^{ \pm}}$коэрцитивными на $H^{1}\left(\Omega^{ \pm}\right)$.

Вместо $H^{\sigma}$ теперь нужны более общие пространства $H_{p}^{\sigma}$ бесселевых потенциалов и $B_{p}^{\sigma}$ Бесова, где $1<p<\infty$; при $p=2$ они совпадают с $H^{\sigma}$. См., 
например, [3] или [4]. Пусть сначала граница замкнута - не имеет края. Тогда решения однородной системы $L u=0$ в $\Omega^{ \pm}$можно искать в пространствах $H_{p}^{1 / 2+s+1 / p}\left(\Omega^{ \pm}\right)$или $B_{p}^{1 / 2+s+1 / p}\left(\Omega^{ \pm}\right)$, данные Дирихле задаются в $B_{p}^{1 / 2+s}(\Gamma)$, а данные Неймана - в $B_{p}^{-1 / 2+s}(\Gamma),|s|<1 / 2$ (при других $s$ теряют, вообще говоря, смысл данные Дирихле на липшицевой поверхности Г или $S$ ). Положим $t=1 / p$. Как и в предыдущих работах автора, допустимые точки $(s, t)$ образуют квадрат

$$
Q=\{(s, t):|s|<1 / 2,0<t<1\} .
$$

Задачи Дирихле и Неймана, однозначно разрешимые в его центре $(0,1 / 2)$, остаются однозначно разрешимыми, по крайней мере, при $|s|<\varepsilon$ и $|t-1 / 2|<\delta$ с достаточно малыми положительными $\varepsilon$ и $\delta$ в силу теоремы Шнейберга об экстраполяции обратимости операторов, действующих в интерполяционных шкалах [27]. Никаких дополнительных ограничений на систему при этом накладывать не нужно. Далее, может быть, с некоторым уменьшением $\varepsilon$ и $\delta$, обобщаются утверждения об ограниченности и обратимости операторов

$$
A: B_{p}^{-1 / 2+s}(\Gamma) \rightarrow B_{p}^{1 / 2+s}(\Gamma) \quad \text { и } \quad H: B_{p}^{1 / 2+s}(\Gamma) \rightarrow B_{p}^{-1 / 2+s}(\Gamma) .
$$

Кроме того, ограничены и обратимы операторы $\frac{1}{2} I \pm B$ в $B_{p}^{1 / 2+s}(\Gamma)$ и $\frac{1}{2} I \pm \widehat{B}$ в $B_{p}^{-1 / 2+s}(\Gamma)$. Обобщается и формула (2.6). Все это объяснено в [3] и [4].

Переходя к случаю границы с краем, нужно прежде всего определить пространство $H_{p}^{1 / 2+s+1 / p}\left(\Omega_{0}\right)$. Определим его так же, как в уже рассмотренном случае $s=0, p=2$ : оно будет состоять из функций $u \in L_{p}\left(\Omega_{0}\right)$, принадлежащих $H_{p}^{1 / 2+s+1 / p}$ в $\Omega^{ \pm}$, следы которых на $\Gamma$ (принадлежащие $B_{p}^{1 / 2+s}(\Gamma)$ ) совпадают на $S_{2}$, так что скачок $[u]$ принадлежит $\widetilde{B}_{p}^{1 / 2+s}(S)$. Пространство $B_{p}^{1 / 2+s+1 / p}\left(\Omega_{0}\right)$ определим аналогично. Замечание, сделанное в п. 1 , распространяется, по крайней мере, на пространства $H_{p}^{1}(\Omega)$.

Мы теперь можем рассматривать решения системы $L u=0$ в $H_{p}^{1 / 2+s+1 / p}\left(\Omega_{0}\right)$ или $B_{p}^{1 / 2+s+1 / p}\left(\Omega_{0}\right)$. В задаче Дирихле

$$
g^{ \pm} \in B_{p}^{1 / 2+s}(S) \quad \text { и } \quad[g] \in \widetilde{B}_{p}^{1 / 2+s}(S) .
$$

В задаче Неймана

$$
h^{ \pm} \in B_{p}^{-1 / 2+s}(S) \quad \text { и } \quad[h] \in \widetilde{B}_{p}^{-1 / 2+s}(S),
$$

так как предложение 1.2 обобщается:

Предложение 6.1. Пусть $u$-решение системы $L u=0$ в $\Omega_{0}$, принадлежсащее $H_{p}^{1 / 2+s+1 / p}\left(\Omega_{0}\right)$. Тогда $[T u] \in \widetilde{B}_{p}^{-1 / 2+s}(S)$.

Доказательство при $L u=0$ проводится аналогично доказательству предложения 1.2 (с заменой $L_{2}$ на $L_{p}$ ).

Далее, из приведенных в п. 6.1 утверждений получаются сначала утверждения об ограниченности операторов

$$
A_{S}: \widetilde{B}_{p}^{-1 / 2+s}(S) \rightarrow B_{p}^{1 / 2+s}(S) \quad \text { и } \quad H_{S}: \widetilde{B}_{p}^{1 / 2+s}(S) \rightarrow B_{p}^{-1 / 2+s}(S),
$$

а также операторов

$$
\frac{1}{2} I \pm B_{S}: \widetilde{B}_{p}^{1 / 2+s}(S) \rightarrow B_{p}^{1 / 2+s}(S) \quad \text { и } \quad \frac{1}{2} I \pm \widehat{B}_{S}: \widetilde{B}_{p}^{-1 / 2+s}(S) \rightarrow B_{p}^{-1 / 2+s}(S)
$$


при $|s|<\varepsilon,|t-1 / 2|<\delta$ с достаточно малыми $\varepsilon$ и $\delta$. Теперь заметим, что используемые здесь семейства пространств являются интерполяционными для комплексного метода интерполяции по каждому из индексов. Для семейств $\left\{B_{p}^{1 / 2+s}(S)\right\}$ и $\left\{B_{p}^{-1 / 2+s}(S)\right\}$ это следует из того, что переход от пространств на $\Gamma$ к соответствующим пространствам на $S$ есть ретракция (обратный переход с использованием оператора продолжения есть коретракция). В отношении семейств $\left\{\widetilde{B}_{p}^{1 / 2+s}(S)\right\}$ и $\left\{\widetilde{B}_{p}^{-1 / 2+s}(S)\right\}$ можно использовать теорему о двойственности для комплексного метода интерполяции: эти пространства двойственны соответственно к $B_{p^{\prime}}^{-1 / 2-s}(S)$ и $B_{p^{\prime}}^{1 / 2-s}(S)$ и рефлексивны. См., например, [9, пп. 4.5 и 6.4]. А так как в центре квадрата $Q$ наши операторы обратимы, то можно применить теорему Шнейберга и сделать вывод об обратимости этих операторов при рассматриваемых $(s, t)$, может быть, с уменьшением $\varepsilon$ и $\delta$. Итак, справедлива

Теорема 6.2. Onераторь (6.4) u (6.5) обратимь при $|s|<\varepsilon,|t-1 / 2|<\delta c$ достаточно малыми $\varepsilon$ и $\delta$.

То же верно для оператора (4.3) при $L=\widetilde{L}$. Обобщается и формула $(2.7)$. Это приводит к следующему основному результату.

Теорема 6.3. Существуют такие $\varepsilon>0 u \delta>0$, что при любъх $g^{ \pm} \in$ $B_{p}^{1 / 2+s}(S)$ u $h^{ \pm} \in B_{p}^{-1 / 2+s}(S),|s|<\varepsilon,|t-1 / 2|<\delta$, задачи Дирихле и соответственно Неймана имеют единственные решения в $H_{p}^{1 / 2+s+1 / p}\left(\Omega_{0}\right)$. Кроме того, в таких же пространствах однозначно разрешимы задача $3^{\circ}$ и задача $4^{\circ}$. Пространства $H$ в этих утверждениях можно заменить на аналогичнъце пространства $B$.

Как следствие при улучшении регулярности $g^{ \pm}$и $h^{ \pm}$улучшается регулярность решений - в указанных пределах.

6.2. Перейдем к спектральным задачам.

Теорема 6.4. Корневые функиии оператора $A_{S}$ принадлежсат объединению пространств $B_{p}^{1 / 2+s}(S),|s|<\varepsilon,|t-1 / 2|<\delta$. Спектр при этом не зависит от $(s, t)$. B пространствах $B_{p}^{1 / 2+s}(S)$ и $\widetilde{B}_{p}^{-1 / 2+s}(S)$ сохраняются утвержсдения о базисности при $p=2$ в случае формальной самосопряженности оператора $L$, о полноте и суммируемости рядов методом Абеля-Лидского в остальных случаях. Утверждения о базисности и полноте распространяются на промежуточные по s пространства.

Для оператора $H_{S}^{-1}$ справедливы аналогичные результаты с заменой пространств $\widetilde{B}_{p}^{-1 / 2+s}(S)$ и $B_{p}^{1 / 2+s}(S)$ соответственно на $B_{p}^{-1 / 2+s}(S)$ и $\widetilde{B}_{p}^{1 / 2+s}(S)$.

Приведем пояснения. Гладкость собственных функций оператора $A_{S}$ видна из уравнения $A_{S} \psi=\lambda \psi$. Аналогично получается гладкость присоединенных функций. Используя (плотные) вложения наших пространств, убеждаемся, как в [1], что сохраняются утверждения о полноте и независимости спектра от $(s, t)$. Немного расширяется промежуток значений $s$, при которых в случае формально самосопряженного оператора $L$ получается базисность. Суммируемость спектральных разложений методом Абеля-Лидского получается с использованием абстрактной теоремы из [2], а необходимая для этого оценка резольвенты, обобщающая (5.4),

$$
\|\varphi\|_{B_{p}^{1 / 2+s}(S)}+|\lambda|\|\varphi\|_{\widetilde{B}_{p}^{-1 / 2+s}(S)} \leqslant C\|\psi\|_{\widetilde{B}_{p}^{-1 / 2+s}(S)},
$$


получается средствами теории интерполяции опять с использованием теоремы Шнейберга. А именно, сначала получается равномерная по параметру оценка первого слагаемого слева, затем, с использованием уравнения (5.3), оценка второго слагаемого.

Добавочно полнота получается во всех пространствах, отвечающих точкам квадрата $Q$, в которые пространства с установленной полнотой плотно вложены. Плотность вложений имеет место автоматически.

7. Спектральные асимптотики. Здесь мы получим асимптотические формулы для собственных значений оператора $A_{S}$ и, при добавочных предположениях, для собственных значений оператора $H_{S}^{-1}$. См. теорему 7.1 ниже. Оператор $L$ теперь предполагаем формально самосопряженным. Сначала мы приведем результаты для операторов в области с замкнутой границей, ср. [4].

7.1. В работе [7] получена асимптотическая формула для собственных значений интегрального оператора на «почти гладкой» липшицевой поверхности Г - псевдодифференциального оператора отрицательного порядка, ядро которого является сужением на $\Gamma \times \Gamma$ ядра эллиптического псевдодифференциального оператора в $\mathbb{R}^{n}$ на единицу меньшего порядка. Почти гладкой там названа поверхность, бесконечно гладкая вне замкнутого подмножества нулевой меры. Вместо $\mathbb{R}^{n}$ можно рассматривать тор.

Примером как раз является наш оператор $A$, если коэффициенты оператоpa $L$ бесконечно гладкие. В этом случае в качестве оператора на торе берем ньютонов потенциал; его ядро - фундаментальное решение для $L$. Предположение о бесконечной гладкости коэффициентов оператора $L$ в конечном счете снимается аппроксимацией заданных коэффициентов бесконечно гладкими, поэтому будем считать коэффициенты бесконечно гладкими. Оператор $A$ сначала рассматривается в $L_{2}(\Gamma)$.

Для вывода асимптотики используется специальное разбиение поверхности на малые части $U_{j}(j=0, \ldots, K)$. Оператор $A$ записывается в виде $\sum A_{j, k}$, где $A_{j, k}=\theta_{j} A \theta_{k} \cdot$ и $\theta_{j}$ - характеристическая функция части $U_{j}$. Асимптотика в случае оператора $A_{j, j}$, отвечающего гладкой части $U_{j}$ поверхности $\Gamma, j>0$, известна, например, из результатов Бирмана-Соломяка. Ключевым моментом является оценка $s$-чисел остальных операторов $A_{j, k}$. Она имеет вид

$$
s_{l}\left(A_{j, k}\right) \leqslant C \min \left(\varepsilon_{j}, \varepsilon_{k}\right) l^{-1 /(n-1)},
$$

где $\varepsilon_{j}$ оценивается через меру множества $U_{j}$ и стремится к нулю, если эта мера стремится к нулю. Здесь существенно, что для нашего $A$ выполнено исходное предположение о ядре рассматриваемого оператора. Оценка (7.1) позволяет использовать технику возмущений, развитую у Бирмана-Соломяка [10], и получить искомый результат. Детали см. в [7, §4]. Ясно, что результат получается и для операторов вида $\theta A \theta \cdot$, если $\theta$ - характеристическая функция части поверхности Г. Именно этим мы воспользуемся дальше.

Предположение о структуре ядра оператора, указанное выше, не выполнено для оператора $H^{-1}$. Но оценки $s$-чисел вида (7.1) можно получить и в том случае, когда оператор допускает две записи $T_{1} A$ и $A T_{2}$, где $T_{1}$ и $T_{2}$ - ограниченные операторы. Из (2.5) видно, что оператор $H^{-1}$ допускает такие представления. Однако нужна ограниченность и обратимость операторов $\frac{1}{2} I \pm B$ и $\frac{1}{2} I \pm \widehat{B}$ не в указанных в п. 2 пространствах, а в $L_{2}(\Gamma)$. Такие результаты 
существуют в литературе, но получены они (на основе совсем другого подхода к задачам в липшицевых областях) не для всех сильно эллиптических систем. Заведомо они справедливы в случае уравнения Бельтрами-Лапласа [23] и системы Ламе [13], к которым можно прибавить младшие члены. В этих случаях асимптотика получается этим методом и для оператора $H^{-1}$ на почти гладкой поверхности. В остальных случаях получается точная по порядку оценка собственных значений.

То, что операторы $A$ и $H^{-1}$ рассматриваются как действующие в $L_{2}(\Gamma)$, не отражается на собственных функциях и собственных значениях.

Ограничение, состоящее в том, что поверхность Г почти гладкая, в случае оператора $A$ сняли Розенблюм и Тащиян [26].

7.2. Перейдем к операторам на незамкнутой поверхности. Пусть

$$
A_{S} \psi=\lambda \psi \text {. }
$$

Здесь сначала $\psi \in \widetilde{H}^{-1 / 2}(S)$. Но вместе с левой частью $\psi$ принадлежат $H^{1 / 2}(S)$. Тогда $\psi$ принадлежит промежуточным пространствам $\widetilde{H}^{s}(S)=H^{s}(S),|s|<1 / 2$, в частности, $L_{2}(S)$. Это позволяет, используя продолжение функций нулем, переписать равенство (7.2) в виде

$$
\theta A \theta \psi=\lambda \psi
$$

где $\theta$ - характеристическая функция для $S$. И обратно, из (7.3) следует (7.2).

Приведем окончательный результат. Собственные значения $\lambda_{j}\left(A_{S}\right)$ оператора $A_{S}$ нумеруются в порядке невозрастания с учетом кратностей.

Теорема 7.1. Имеет место формула

$$
\lambda_{j}\left(A_{S}\right)=C_{A_{S}} j^{-1 /(n-1)}+o\left(j^{-1 /(n-1)}\right),
$$

¿əe

$$
C_{A_{S}}^{n-1}=(2 \pi)^{-(n-1)} \iint_{T^{*} S} n_{\alpha}\left(x^{\prime}, \xi^{\prime}\right) d x^{\prime} d \xi^{\prime},
$$

$\alpha\left(x^{\prime}, \xi^{\prime}\right)$ - главный символ оператора $A$ u $n_{\alpha}\left(x^{\prime}, \xi^{\prime}\right)$ - число его собственных значений, больших 1 .

Для оператора $H_{S}^{-1}$ справедлив аналогичный результат в случае почти гладкой поверхности $\Gamma$, если оператор $L$ скалярный или если это матричный оператор с оператором Ламе в старией части.

Кокасательное расслоение и символ на липшицевой поверхности понимаются формально, но кокасательные пространства и символ определены почти всюду на $S$ и формуле (7.5) можно придать смысл как в случае почти гладкой поверхности (см. [7]), так и в общем случае (см. [26]).

На вычислении символов операторов $A$ и $H^{-1}$ (в гладком случае) останавливаться не будем, см. указания в [4].

В заключение сделаем три замечания.

1. Мы считали, что обе области $\Omega^{ \pm}$лежат на торе. Вместо тора в принципе можно рассмотреть содержащую Г область с гладкой границей и, например, однородным условием Дирихле на ней или более общее гладкое многообразие с гладким краем или без края. Но можно (и полезно) рассматривать $\mathbb{R}^{n}$; тогда надо выбирать подходящие условия в $\Omega^{-}$на бесконечности, что отражается, конечно, на выборе пространств. См. [11]. 
2. Если не предполагать, что коэрцитивность форм $\Phi_{\Omega^{ \pm}}$имеет место в усиленной форме, то вместо теорем об однозначной разрешимости задач или об обратимости операторов получаются результаты об их фредгольмовости с нулевым индексом. Эти обобщения не требуют большого труда, но приводят к усложнениям формулировок; мы избегали на этом останавливаться.

3. В текст этой работы внесены добавления перед ее сдачей в печать: добавлены смешанные задачи и спектральные асимптотики.

Автор искренне благодарит Т. А. Суслину: она ознакомилась с работой и прислала список ценных редакционных замечаний.

\section{ЛитерАтУРА}

[1] М. С. Агранович, Регулярность вариащионных решений линейных граничных задач в липшицевых областях, Функц. анализ и его прил., 40:4 (2006), 83-103.

[2] М. С. Агранович, Спектральные задачи в липшицевых областях для силъно эллиптических систем в банаховых пространствах $H_{p}^{\sigma}$ u $B_{p}^{\sigma}$, Функц. анализ и его прил., 42:4 (2008), 2-23.

[3] М. С. Агранович, Операторы типа потенциала и задачи сопряжсения для силъно эллиптических систем 2-го порядка в областях с липшицевой гранищей, Функц. анализ и его прил., 43:3 (2009), 3-25.

[4] М. С. Агранович, Спектральные задачи в липшицевых областях, Современные проблемы математики, Фундаментальные направления, т. 39 (в печати).

[5] М. С. Агранович, Смешанные задачи в липшищевой области для сильно эллиптических систем 2-го порядка, Функц. анализ и его прил., 45 (в печати).

[6] M. S. Agranovich, Strongly elliptic second order systems with spectral parameter in transmission conditions on a nonclosed surface, in: Operator Theory: Advances and Applications, vol. 164, Birkhäuser, Basel, 2006, 1-21.

[7] М. С. Агранович, Б. А. Амосов, Оценки s-чисел и спектралъные асимптотики для интегральных операторов типа потенциала на негладких поверхностях, Функц. анализ и его прил., 30:2 (1996), 1-18.

[8] M. S. Agranovich, B. Z. Katsenelenbaum, A. N. Sivov, N. N. Voitovich, Generalized Method of Eigenoscillations in Diffraction Theory, Wiley-VCH, Berlin, 1999. (Пepeработанное английское издание книги [31].)

[9] Й. Берг, Й. Лёфстрём, Интерполячионные пространства. Введение, Мир, М., 1980.

[10] М. Ш. Бирман, М. З. Соломяк, Спектралъная асимптотика негладких эллиптических операторов, I, Труды ММО, 27 (1972), 3-52.

[11] M. Costabel, M. Dauge, On representation formulas and radiation conditions, Math. Methods Appl. Sci., 20:2 (1997), 133-150.

[12] M. Costabel, E. Stephan, An improved boundary element Galerkin method for threedimensional crack problems, Integral Equations and Operator Theory, 10:4 (1987), $467-504$.

[13] B. E. J. Dahlberg, C. E. Kenig, G. C. Verchota, Boundary value problems for systems of elastostatics in Lipschitz domains, Duke Math. J., 57:3 (1988), 795-818.

[14] R. Duduchava, D. Natroshvili, Mixed crack type problem in anisotropic elasticity, Math. Nachr., 191 (1998), 83-107.

[15] Р. В. Дудучава, Д. Г. Натрошвили, Е. М. Шаргородский, Граничнъе задачи математической теории трещин, Труды Института прикладной математики им. И. Н. Векуа Тбилисского унив., 39 (1990), 68-84.

[16] R. Duduchava, W. L. Wendland, The Wiener-Hopf method for systems of pseudodifferential equations with an application to crack problems, Integral Equations Operator 
Theory, 23:3 (1995), 294-335.

[17] Г. И. Эскин, Краевые задачи для эллиптических псевдодифференциалъных уравнений, Наука, М., 1973.

[18] P. Grisvard, Elliptic Problems in Nonsmooth Domains, Pitman, Boston, 1985.

[19] G. C. Hsiao, E. P. Stephan, W. L. Wendland, An integral equation formulation for a boundary value problem of elasticity in the domain exterior to an arc, in: Lecture Notes in Math., vol. 1121, Springer-Verlag, Berlin, 1983, 153-165.

[20] G. C. Hsiao, W. L. Wendland, Boundary Integral Equations, Springer-Verlag, Berlin, 2008.

[21] W. McLean, Strongly Elliptic Systems and Boundary Integral Equations, Cambridge Univ. Press, Cambridge, 2000.

[22] S. E. Mikhailov, Traces, extensions, co-normal derivatives and solution regularity of elliptic systems with smooth and non-smooth coefficients, http://arxiv.org/abs/ 0906.3875 .

[23] M. Mitrea, M. Taylor, Boundary layer methods for Lipschitz domains in Riemannian manifolds, J. Funct. Anal., 163:2 (1999), 181-251.

[24] J. Nečas, Les méthodes directes en théorie des équations elliptiques, Masson, Paris, 1967.

[25] O. A. Oleinik, A. S. Shamaev, G. A. Yosifian, Mathematical Problems in Elasticity and Homogenization, North Holland, Amsterdam, 1992.

[26] G. Rozenblum, G. Tashchiyan, Eigenvalue asymptotics for potential type operators on Lipschitz surfaces, Russian J. Math. Phys., 13:3 (2006), 326-339.

[27] И. Я. Шнейберг, Спектралъные свойства линейных операторов в интерполяционных семействах банаховых пространств, Матем. исслед., 9:2 (1974), 214-227.

[28] E. Stephan, Boundary integral equations for mixed boundary value problems, screen and transmission problems in $\mathbb{R}^{3}$, Habilitationsschrift, Darmstadt, THD-preprint 848, 1984 .

[29] E. Stephan, A boundary integral equation method for three-dimensional crack problems in elasticity, Math. Methods Appl. Sci., 8:4 (1986), 609-623.

[30] E. Stephan, Boundary integral equations for screen problems in $\mathbb{R}^{3}$, Integral Equations Operator Theory, 10:2 (1987), 236-257.

[31] Н. Н. Войтович, Б. З. Каценеленбаум, А. Н. Сивов, Обобщенный метод собственных колебаний в теории дифракции, с добавлением М. С. Аграновича «Спектральные свойства задач дифракции», стр. 289-416, Наука, М., 1977.

Московский институт электроники и математики e-mail: magran@orc.ru
Поступило в редакцию 28 апреля 2010 г. 